\title{
Unusual massive gastric mucosal bleeding in a patient with dengue
}

\author{
C N Wijekoon ${ }^{1}$, A S Dassanayake ${ }^{1}$, P W M C S B Wijekoon ${ }^{2}$, V Ratnamalala ${ }^{3}$
}

Journal of the Ceylon College of Physicians, 2013, 44, 42-44

\section{Introduction}

Dengue has become one of the most important communicable diseases in Sri Lanka. 44,410 cases of dengue have been reported in Sri Lanka in $2012^{1}$. Gastrointestinal ( $\mathrm{Gl}$ ) bleeding is a well recognised complication of dengue. However severe GI bleeding is usually seen in patients who develop shock, multiorgan failure and disseminated intravascular coagulation (DIC) or in those who have taken nonsteroidal anti-inflammatory drugs (NSAIDs) ${ }^{2}$. Here we report a patient with dengue who had massive $\mathrm{GI}$ bleeding in the absence of any of the above contributing factors and we describe how timely administered conservative management could be life saving.

\section{Case report}

In November 2012, a previously healthy 17 year old boy was admitted to a private hospital in Wattala on the third day of a febrile illness. He also complained of severe headache, loose motions and faintishness. He did not have vomiting or bleeding manifestations at the time of admission. Before coming to the hospital he has taken treatment from a general practitioner. Prescribed drugs were paracetamol, domperidone, coamoxiclav and fexofenadine. He gave a history of allergy to ibuprofen and had not taken any NSAIDs or steroids in the recent past. He did not have a history of peptic ulcer disease and has not been on anticoagulant therapy. He had never smoked or consumed alcohol.

The clinical examination on admission revealed an alert but ill patient with mild to moderate dehydration. His temperature was $101.1^{\circ} \mathrm{F}$. There was no pallor, jaundice, skin rashes or flushing. His blood pressure (BP) was $100 / 70 \mathrm{mmHg}$ with no postural drop. Pulse rate was 80 beats per minute (bpm) and the pulse volume was normal. There was epigastric tenderness. The liver and spleen were not palpable and there was no free fluid in the abdomen. Respiratory

\footnotetext{
${ }^{1}$ Faculty of Medicine, University of Kelaniya, Sri Lanka.

${ }^{2}$ Faculty of Medical Sciences, University of Sri Jayawardenapura, Sri Lanka.

${ }^{3}$ Colombo North Teaching Hospital, Ragama, Sri Lanka.
}

system was normal with no evidence of pleural effusions. On admission, platelet count (PC) was $95,000 / \mathrm{mm}^{3}$, white cell count (WCC) was $2600 / \mathrm{mm}^{3}$. Hematocrit was $46.7 \%$ with hemoglobin of $15.5 \mathrm{~g} / \mathrm{dl}$. Liver transaminases were normal [aspartate aminotransferase (AST)-47U/l, alanine aminotransferase (ALT) - 43U/l]. Dengue NS 1 antigen test was positive. It was done by SD BIOLINE Dengue NS $1 \mathrm{Ag}$ rapid test which is an in-vitro immunochromatographic one step assay for qualitative determination (sensitivity$92.8 \%$; specificity-98.4\%).

Management at this stage consisted of fluid resuscitation with crystalloids and oral rehydration solution, symptomatic therapy with paracetamol and omeprazole and close monitoring. After resuscitation, initially high hematocrit dropped to $40.9 \%$ with hemoglobin of $14.2 \mathrm{~g} / \mathrm{dl}$. BP had risen to $110 / 70 \mathrm{mmHg}$ and pulse rate was $80 \mathrm{bpm}$. Serial blood counts over the next 24 hours revealed further reduction of PC and WCC but the hematocrit remained around $40 \%$.

Late on the $4^{\text {th }}$ day of fever PC and WCC were $40,000 / \mathrm{mm}^{3}$ and $1400 / \mathrm{mm}^{3}$, respectively and hematocrit was $39.8 \%$. Patient was febrile but was haemodynamically stable with adequate urine output. There were no features of fluid leakage. He had epigastric tenderness but there was no vomiting, hepatomegaly or bleeding manifestations. Few hours later, patient suddenly vomited $200-250 \mathrm{ml}$ of fresh blood. The blood count done at the onset of bleeding showed a PC of $31,000 / \mathrm{mm}^{3}$. Liver transaminases have increased (AST - $346 \mathrm{U} / 1$; ALT - $178 \mathrm{U} / 1$ ) but prothrombin time (PT) and activated partial thromboplastin time (APTT) were normal.

Over the next 48 hours, patient had several episodes of massive haematamesis and melaena periodically. Persistent tachycardia above $100 \mathrm{bpm}$ continued throughout the $5^{\text {th }}, 6^{\text {th }}$ and $7^{\text {th }}$ days and haematocrit continued to drop in spite of blood transfusions indicating continued active bleeding. With resuscitation systolic BP was maintained between 90$100 \mathrm{mmHg}$. There was no narrowing of pulse pressure (i.e. $\leq 20 \mathrm{mmHg}$ ) at any point. Adequate urine output was maintained with no significant positive or negative balance. Patient was afebrile from the $6^{\text {th }}$ day onwards. Lowest PC was recorded in the afternoon of the $5^{\text {th }}$ day and it was $19,300 / \mathrm{mm}^{3}$. The lowest recorded hemoglobin level was $7.1 \mathrm{~g} / \mathrm{dl}$. On the $6^{\text {th }}$ day APTT 
was prolonged but the PT was normal. Highest levels of transaminases were recorded on the $8^{\text {th }}$ day (AST $458 \mathrm{U} / 1$; ALT - $341 \mathrm{U} / 1$ ) when patient was recovering from bleeding and serum bilirubin never went up. His serum creatinine and electrolytes remained normal. On the $6^{\text {th }}$ day serum ionized calcium was low $(0.9 \mathrm{mmo} 1 / 1)$. But repeated ultrasound scan of the abdomen and chest did not reveal free fluids at any point.

Upper gastrointestinal endoscopy (UGIE) was considered on the $5^{\text {th }}$ day but the patient vomited fresh blood just before the procedure and it was abandoned considering the risks associated with proceeding further. The UGIE was performed later on, on the $7^{\text {th }}$ day when bleeding was settling. It revealed a diffuse gastropathy; altered blood was present in the stomach; there were no ulcers or erosions; the duodenum and the esophagus were normal.

The patient was managed medically in the intensive care unit. Blood and blood components were transfused and supportive therapy was given. The transfusion requirements were guided by the patient's clinical condition. In total, patient received 4 units of fresh whole blood and 13 units of packed red cells. He also received 17 units of platelets and 17 units of fresh frozen plasma. Intravenous normal saline was administered through out and the rate was adjusted according to the clinical parameters and the fluid balance status. During the period of active bleeding he was treated with esomeprezole $80 \mathrm{mg}$ intravenously as a stat dose followed by continuous intravenous infusion of $8 \mathrm{mg} / \mathrm{hour}$, intravenous tranexamic acid $1 \mathrm{~g}$ 8hourly and intravenous vitamin K $10 \mathrm{mg}$ daily. Intravenous calcium gluconate was given to correct hypocalcaemia.

Apart from a transient bradycardia on the $9^{\text {th }}$ and $10^{\text {th }}$ days the recovery phase was uneventful. The patient was discharged from the hospital on the $13^{\text {th }}$ day. On discharge he had a PC of $230,000 / \mathrm{mm}^{3}$, WCC of $5,200 / \mathrm{mm}^{3}$ and hemoglobin of $13.1 \mathrm{~g} / \mathrm{d} 1$. AST was $116 \mathrm{U} / 1$ and ALT was $250 \mathrm{U} / 1$. On subsequent followup after 2 weeks he was asymptomatic and the liver transaminases and the blood counts were within normal limits.

\section{Discussion}

Dengue is a complex and dynamic disease with a clinical spectrum that includes both severe and nonsevere clinical manifestations ${ }^{3}$. The course of dengue illness classically has three phases, namely the febrile phase, the critical phase and the recovery phase. Plasma leakage leading to shock, severe haemorrhage and severe organ impairment are the complications that could occur in the critical phase ${ }^{2}$.

Major bleeding in patients with dengue, is almost always associated with shock since this, in combination with hypoxia and acidosis, can lead to multiple organ failure and advanced DIC. Massive GI bleeding may occur without prolonged shock when NSAIDs or steroids have been taken or with pre-existing peptic ulcer disease or with anticoagulant therapy ${ }^{2}$.

There are only a few reports on the endoscopic findings in dengue patients with GI bleeding. In Taiwan endoscopic examination of 97 dengue patients with GI bleeding revealed hemorrhagic gastritis in $67 \%$, gastric ulcer in $57.7 \%$, duodenal ulcer in $26.8 \%$, and esophageal ulcer in $3.1 \%{ }^{4}$. In another study, among 26 dengue patients with $\mathrm{GI}$ bleeding, most had gastric or duodenal ulcers ${ }^{5}$.

Prophylactic platelet transfusions to prevent any form of bleeding have not been shown to be effective in dengue even in the presence of severe thrombocytopenia ${ }^{6}$. Blood transfusion is life-saving and should be given as soon as severe bleeding is suspected or recognized with caution because of the risk of fluid overload $^{2}$. There is little evidence to support the practice of transfusion of platelet concentrates and fresh-frozen plasma (FFP) when massive bleeding occurs in patients with dengue ${ }^{2}$. Nevertheless coadministration of higher ratios of plasma and platelets along with packed red blood cells ( $p R B C$ ) help to prevent coagulopathy and this approach is associated with improved survival in patients with massive hemorrhage due to any cause ${ }^{7}$. There is some evidence for the usefulness of recombinant factor VII as adjunctive therapy in severe bleeding due to dengue in children ${ }^{8}$.

Evidence is lacking for the effectiveness of endoscopic therapy in dengue patients with GI bleeding. Chiu YC et al have reported that, in dengue patients with peptic ulcer, endoscopic injection therapy is not effective $\mathrm{e}^{4}$. In this study, after endoscopy patients who had injection therapy required more transfusions with $\mathrm{pRBC}$ and FFP than the control group.

The patient we report here had severe dengue according to the World Health Organization classification ${ }^{2}$. However his clinical course was unusual due to several reasons. Prior to the onset of major GI bleeding patient's haemodynamic status and hematocrit were stable. He did not have any feature of plasma leakage to indicate that he was in critical phase. The only warning sign of severe dengue he had prior to the onset of bleeding was epigastric tenderness. 
The bleeding started at a relatively high platelet count and in the absence of evidence of shock, coagulopathy or DIC. Patient did not have any other risk factors for GI bleeding such as recent use of NSAIDs or steroids, anticoagulant use or history of peptic ulcer disease. UGIE did not reveal peptic ulcers or erosions and the massive bleeding which required 17 units of blood as replacement therapy was purely due to diffuse gastropathy. The slow oozing leading to collection of blood in the stomach manifested as periodic massive haematamesis and melaena. It was probably the slowness of the mucosal oozing which prevented him from going in to acute shock secondary to bleeding.

This case is a good example for the complex and unpredictable nature of dengue and illustrates that massive GI bleeding can occur even in the absence of generally identified risk factors. However when the bleeding started the platelet count was rapidly dropping despite the fact that the absolute count was relatively high. These findings highlight the need for close monitoring of all such patients even if the other warning signs are minimal. This case also shows that in patients with dengue, the usefulness of UGIE at the time of acute GI bleeding is limited. Benefits are minimal in cases like this as it cannot offer any effective therapy and the risks are high as any mucosal damage can lead to further bleeding because of platelet dysfunction. Furthermore this case highlights that timely and correctly administered conservative treatment with transfusion of blood components and supportive therapy alone is life saving for such patients even if the bleeding is massive.

\section{References}

1. Epidemiology Unit, Ministry of Health, Sri Lanka. http:// www.epid.gov.lk/web/index.php?option=com_casesanddeaths \& Itemid=448 \& lang=en\# (cited February 20, 2013).

2. World Health Organization and the Special Programme for Research and Training in Tropical Diseases. Dengue guidelines for diagnosis, treatment, prevention and control. New Edition. 2009. http://whqlibdoc.who.int/publications/ 2009/9789241547871_eng.pdf (cited February 20, 2013).

3. Rigau-Pérez JG, Clark GG, Gubler DJ, Reiter P, Sanders EJ, Vorndam AV. Dengue and dengue haemorrhagic fever. Lancet 1998; 352: 971-7.

4. Chiu YC, Wu KL, Kuo $\mathrm{CH}$, et al. Endoscopic findings and management of dengue patients with upper gastrointestinal bleeding. Am J Trop Med Hyg 2005; 73(2): 441-4.

5. Tsai CJ, Kuo $\mathrm{CH}$, Chen PC, Changcheng CS. Upper gastrointestinal bleeding in dengue fever. Am J Gastroenterol 1991; 86(1): 33-5.

6. Lum LC, Abdel-Latif Mel-A, Goh AY, Chan PW, Lam SK. Preventive transfusion in dengue shock syndrome - is it necessary? Journal of Pediatrics 2003;143: 682-4.

7. Young PP, Cotton BA, Goodnough LT. Massive transfusion protocols for patients with substantial hemorrhage. Transfus Med Rev 2011; 25(4): 293-303.

8. ChuansumritA, Wangruangsatid S, Lektrakul Y, Chua MN, Zeta Capeding MR, Bech OM. Control of bleeding in children with Dengue hemorrhagic fever using recombinant activated factor VII: a randomized, double-blind, placebo-controlled study. Blood Coagul Fibrinolysis 2005; 16(8): 549-55. 